\title{
Phthalocyanine and encapsulated polyaniline nanoparticles as fillers for dielectric elastomers
}

\author{
Dorina M. Opris ${ }^{\mathrm{a}^{*}}$, Daniel Crespy ${ }^{\mathrm{b}}$, Christiane Löwe ${ }^{\mathrm{a}}$, Martin Molberg ${ }^{\mathrm{a}}$, Frank Nüesch ${ }^{\mathrm{a}}$ \\ ${ }^{a}$ Empa, Swiss Federal Institute for Materials Testing and Research, Überlandstrasse \\ 129, CH-8600 Dübendorf, Switzerland \\ ${ }^{b}$ Empa, Swiss Federal Institute for Materials Testing and Research, Lerchenfeldstrasse 5, CH-9014 \\ St. Gallen, Switzerland
}

\begin{abstract}
The dielectric constant $(\varepsilon)$ of a polymer can significantly be increased by blending it with conducting fillers. Given our interest in developing highly efficient and long-lasting actuators for muscle replacement, we set out to explore all key issues which could help to reduce the required voltage and at the same time ensure long term stability. The presentation describes experiments which prove that the water content in carboxylic acid-decorated phthalocyanines (Pcs), commonly falsely referred to oligo-Pcs, is a critical factor determining the absolute value of $\varepsilon$. Several publications on $\varepsilon$ values of these oligo-Pcs led to contradicting conclusions because the effect of water was not sufficiently considered. The water content is relevant because o-Pcs are often used as fillers to increase $\varepsilon$ of polymer matrices. This presentation also describes an experimental evaluation on whether or not as-prepared polyaniline (PANI) and poly(divinyl benzene)encapsulated (PDVB) PANI can be reasonably used as high $\varepsilon$ fillers in matrix materials. For this purpose several blends with polystyrene-polybutadiene block copolymer gels (PS- $b$-PB) and polydimethyl siloxane (PDMS) were prepared and their dielectric properties investigated. The former part of this presentation has in part already been published (D. M. Opris et al. Chem. Mater. 20(21), 6889-6896, 2008), the latter is completely new.
\end{abstract}

Keywords: phthalocyanine, polyaniline, miniemulsion polymerization, encapsulation, dielectric elastomers, electroactive polymers

\section{INTRODUCTION}

Dielectric elastomer actuators (DEA) came recently more into the focus of both basic and applied research because of their fascinating potential applications including mobile robots, pumps and valves, loudspeakers, sensors, lenses, and perhaps most importantly artificial muscles. ${ }^{1}$ DEA are made from a thin elastic film sandwiched between two compliant electrodes. When an electric voltage is applied, an electrostatic pressure is acting on the film compressing it in thickness while elongating it in plane. This way, electrical energy is converted into mechanical work. However, a broad application of DEA is hindered by the required high driving voltage which is in the $\mathrm{kV}$ regime. ${ }^{2}$ In order to lower this voltage without having a negative impact on elongation, the films should be as thin as possible, the polymers' elastic modulus as low as possible, and its $\varepsilon$ as high as possible. ${ }^{3}$ To achieve matrices with high $\varepsilon$, highly polarizable additives [e.g. phthalocyanines (Pcs), ceramics] as well as conductive fillers such as PANI, polythiophene, C-black, carbon nanotubes, metals have been incorporated into elastomer matrices. ${ }^{4}$

This contribution describes the three fillers with high $\varepsilon$ Pc, PANI particles, and PDVB encapsulated PANI which were tested to increase the matrices' effective $\varepsilon$. Pc was selected because of its outstandingly high $\varepsilon\left(>10^{3}\right){ }^{5}$ It was hoped that low volume fractions may be sufficient to achieve the desired dielectric properties and blending would therefore not deteriorate the elastic properties of the matrix. Commercial PANI base (molar mass 5,000 g/mol) was selected because it is well known that it can significantly increase $\varepsilon$ of a polymer when it is blended close to percolation. Polyurethanes are a commonly used matrix material for such purpose. Here PS- $b$-PB gels were used as novel matrix material because it was expected that this thermoplastic gel could easily be blended without loosing its mechanical properties. PANI particles are known for their high agglomeration tendency. In order to prevent this from happening as far as possible, it was tried to coat PANI nanoparticles with insulating PDVB. The encapsulation process will be described as well as the blending of the encapsulation product into both PS- $b$-PB and polydimethyl siloxane (PDMS).

Electroactive Polymer Actuators and Devices (EAPAD) 2009, edited by Yoseph Bar-Cohen, Thomas Wallmersperger, Proc. of SPIE Vol. 7287, 72870L · (C) 2009 SPIE · CCC code: 0277-786X/09/\$18 · doi: 10.1117/12.815790 


\section{PHTHALOCYANINES AS HIGH DIELECTRIC CONSTANT FILLERS}

So-called o-Pcs were often used as fillers to increase $\varepsilon$ of a polymer. Their blends into polyurethanes led to very high $\varepsilon$ materials. ${ }^{6} \mathrm{~A}$ volume fraction as low as $3.5 \%$ of o-CuPc decorated with a poly(acrylic acid) was sufficient to increase $\varepsilon$ of a polyurethane matrix to $4186(\tan \delta \sim 0.5)$ at $20 \mathrm{~Hz}$. A strain of $8.3 \%$ was observed under a field of $11.5 \mathrm{~V} / \mu \mathrm{m}$ at 1 $\mathrm{Hz}^{6}{ }^{6}$ o-Pcs are usually synthesized from pyromellitic anhydride or 1,2,4,5-tetracyanobenzene either in solution or in melt. ${ }^{7}$ In a long term project at EMPA, which deals with dielectric elastomers for actuators, it became evident that the literature describing the dielectric properties of o-Pc's is rather confusing, partially even contradicting. In order to provide a sound basis for the reproducible and reliable preparation of elastomers for actuator technology, we set out to explore the reasons for these conflicting reports. In a recent publication we described that some of the o-Pc's reported in the literature consist basically of monomeric species. ${ }^{8}$ This was concluded from a comparison of the products of a literature procedure for o-Pcs with those of a procedure which intrinsically gave strictly monomeric Pc. Whereas the former products when measured at $20^{\circ} \mathrm{C}$ and in air gave irreproducible $\varepsilon$ values the latter products gave fully reproducible $\varepsilon$-values if the water content was strictly controlled. To illustrate the irreproducibility of the former products Figure 1 shows the dielectric properties of three independent preparations which macroscopically were all performed so as to give exactly the same product. The samples show grossly different absolute $\varepsilon$ values at frequency dependence.

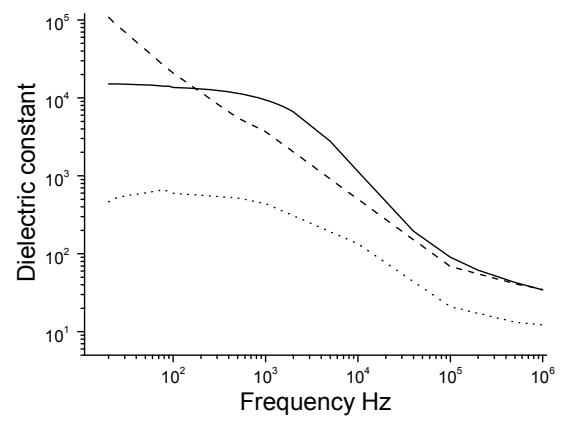

Fig. 1. Dielectric constant of supposedly identical samples without controlling the water content: sample 1 (full line), sample 2 (dashed line), sample 3 (dotted line) as function of frequency at room temperature.

According to the literature this difference is due to the polymerization degree and doping by oxygen. ${ }^{5}$ Increasing bathochromic shifts of the long wavelength UV absorption of o-Pcs with increasing numbers of conjugated Pc units is a well-known effect. ${ }^{9}$ If the differences in the dielectric constant were due to the polymerization degree, such a bathochromic shift was to be expected for the samples with higher dielectric constant. However, the UV spectra in aqueous $\mathrm{NaOH}$ of different samples exhibited the same absorption band at $\lambda=680 \mathrm{~nm}$, typical for monomeric phthalocyanines. In addition to this, another absorption band at $\lambda=830 \mathrm{~nm}$ was observed. Based on mass spectrometry the absorption at $\lambda=830 \mathrm{~nm}$ was assigned to the dimer. ${ }^{8}$

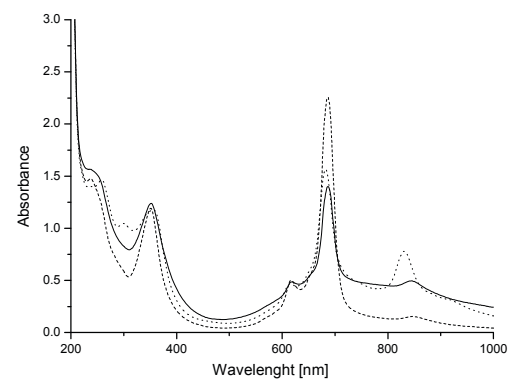

Fig. 2. UV-vis spectra of different samples of "o-Pcs" in aqueous $\mathrm{NaOH}$ : sample 1 (full line), sample 2 (dashed line), sample 3 (dotted line). The sample numbers refer to those in Figure 1. 
Because of the almost identical appearance of the UV spectra shown in Figure 2 with those of "o-Pcs" reported in the literature, it is concluded that the latter were recorded from basically monomeric structures. This was finally clarified by the synthesis of the strictly monomeric Pc 1 (Scheme 1). ${ }^{8,10}$

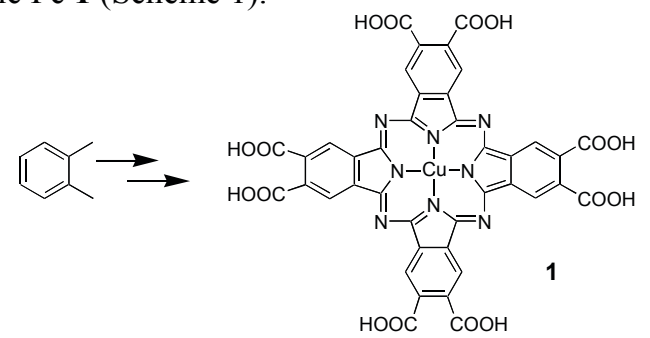

Scheme 1. The synthesis of monomeric phthalocyanine 1 starting from o-xylene. ${ }^{8}$

The dielectric properties of pressed pellets of $\mathbf{1}$ were investigated as a function of frequency. It was observed that when exposing powdered 1 to water vapor, a high increase of $\varepsilon$ was found. The low frequency dielectric constant of the pellets raised by four orders of magnitude from $\sim 12$ to $>10^{5}$ when increasing the water content by a factor of 2 from $6.4 \%$ to $14.7 \%(\mathrm{w} / \mathrm{w})$, respectively (Figure 3$)$.

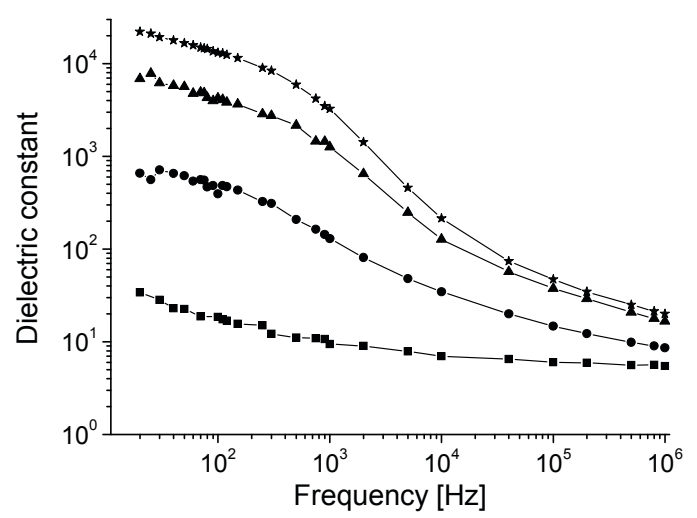

Fig. 3. Dielectric constants of $\mathbf{1}$ at room temperature as function of frequency under different relative humidity conditions $0 \%(\boldsymbol{\bullet}), 20 \%(\bullet), 43 \%(\boldsymbol{\Delta}), 52 \%(*)$ (taken from reference 8$)$.

This strong $\varepsilon$ dependence on the water content causes problems in obtaining blends with constant water content and thus renders fabrication of actuators with long-term stability complicated. A possible way to overcome this is to replace the water with the less volatile imidazole. This compound forms similar hydrogen bond networks like water and its transport properties in the liquid state are also similar to that of water. ${ }^{11}$ Work in this direction is underway.

\section{PANI AND PDVB ENCAPSULATED PANI}

Conducting polymers found a large number of applications including sensors and actuators, antistatic formulations, electronic devices, and conducting paints to name a few. It is well known that the dielectric constant of a matrix can be significantly increased by conductive fillers near the percolation threshold. ${ }^{12}$ PANI has the advantage of combining easy preparation from low cost materials with good environmental stability. In addition to this, it has a low elastic modulus and thus will not change the blend's modulus very much. However, it is very poorly soluble and difficult to process. Its solubility and thus its compatibility with matrices can be much increased by either covalently attaching long alkyl chains to the phenyl rings or by protonating its base form with long chain sulphonic acids. ${ }^{13}$ This latter process converts PANI into a "hairy" polyelectrolyte. Thus PANI can be made soluble in common organic solvents including toluene, tetrahydrofurane, xylene, etc. and has often been used to increase $\varepsilon$ of a polymer matrix. Zhang blended PANI into a polyurethane matrix and obtained an increase of the dielectric constant. ${ }^{14}$ 
Based on these findings, we tried to prepare blends of PANI into a PS- $b$-PB. In order to increase the solubility of PANI into toluene, PANI base (molar mass $5,000 \mathrm{~g} / \mathrm{mol}$ ) was doped with dodecylbenzene sulfonic acid (DBSA). ${ }^{13}$ Composites of PS- $b$-PB with different concentration of doped PANI were produced. The films were prepared by casting from a toluene solution at room temperature. The dielectric constant increased significantly with PANI concentration. Certain composites showed $\varepsilon>1000$ (Figure 4). However, the dielectric loss increased significantly (Figure 4), so that such materials were obviously too conductive and could not reasonably be used as actuators. It should be also mentioned that processing has very much influence on the dielectric properties of the blends. For example, when the solvent was removed fast, the blends showed a $\varepsilon$ lower by a factor of $10^{2}$.
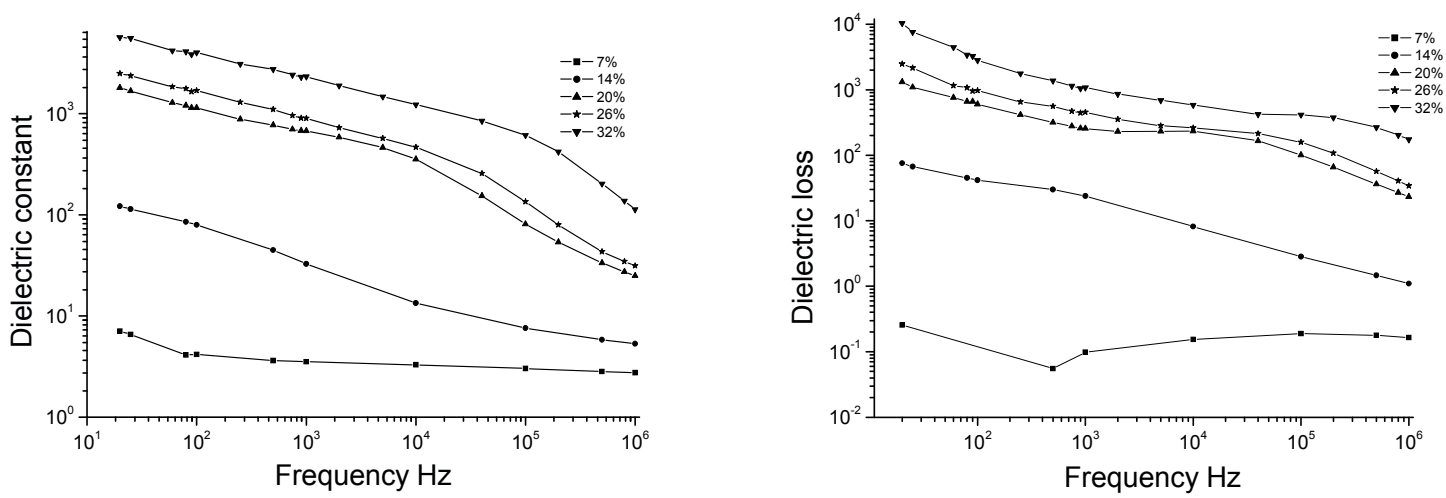

Fig. 4. Dielectric constant (left) and dielectric loss (right) as a function of frequency measured for composites with different concentrations of PANI/DBSA 1:0.5.

Agglomerated particles due to incompatibility of the conductive filler to the matrix, may induce electrical breakthroughs due to field enhancement between the particles. ${ }^{12}$ To avoid such a situation it was of primary importance to fabricate a composite with well dispersed conducting particles. A way to increase the compatibility of PANI with a matrix is to encapsulate it into insulating polymers. This can be achieved using miniemulsion polymerization technique. They produce functional polymer colloids with size ranging from 30 to $500 \mathrm{~nm}$. It is presently widely used to encapsulate various materials. ${ }^{15}$ In order to achieve PANI nanoparticles encapsulated into a PDVB shell, the PANI particles were prepared as follows using a modified literature procedure. ${ }^{16}$ Aniline was polymerized in an acidic aqueous solution containing poly(styrenesulfonic acid) as a stabilizer. Stable nanoparticles with sizes around $100 \mathrm{~nm}$ (dynamic light scattering) were obtained as stable dispersions in water. Their incorporation into PDVB was tried by mixing stabilized PANI dispersions in water with divinyl benzene, hexadecane as hydrophobe, initiator AIBN, and surfactant solution. The best surfactant for this system was Tween 80 . Table 1 presents the reaction conditions of two experiments where the amount of the divinyl benzene added to the mixture was varied and the amount of PANI kept constant.

Table 1. Reaction conditions of two miniemulsion experiments. The quantities of chemicals are given in $\mathrm{g}$.

\begin{tabular}{|c|c|c|c|c|c|c|c|}
\hline Sample & Aq. PANI & DVB & Hexadecane & AIBN & Water & Tween 80 & ${\text { Diameter }(\mathrm{nm})^{\mathrm{b}}}^{\mathrm{a}}$ \\
\hline $\mathrm{mp}-1$ & 6.4 & 1 & 0.5 & 0.1 & 18 & 0.1 & 280 \\
\hline $\mathrm{mp}-2$ & 6.4 & 0.75 & 0.25 & 0.075 & 18 & 0.1 & 200 \\
\hline
\end{tabular}

${ }^{\mathrm{a}}$ For all miniemulsion experiments PANI dispersions in water were used. Its synthesis is described in the experimental part. ${ }^{b}$ Measured by SEM

These polymerizations produced nanometer-sized particles. According to scanning electron microscopy (SEM) (Figure 5) the average diameters are 280 for the sample $\mathrm{mp}-1$ and $200 \mathrm{~nm}$ for $\mathrm{mp}-2$, respectively. 

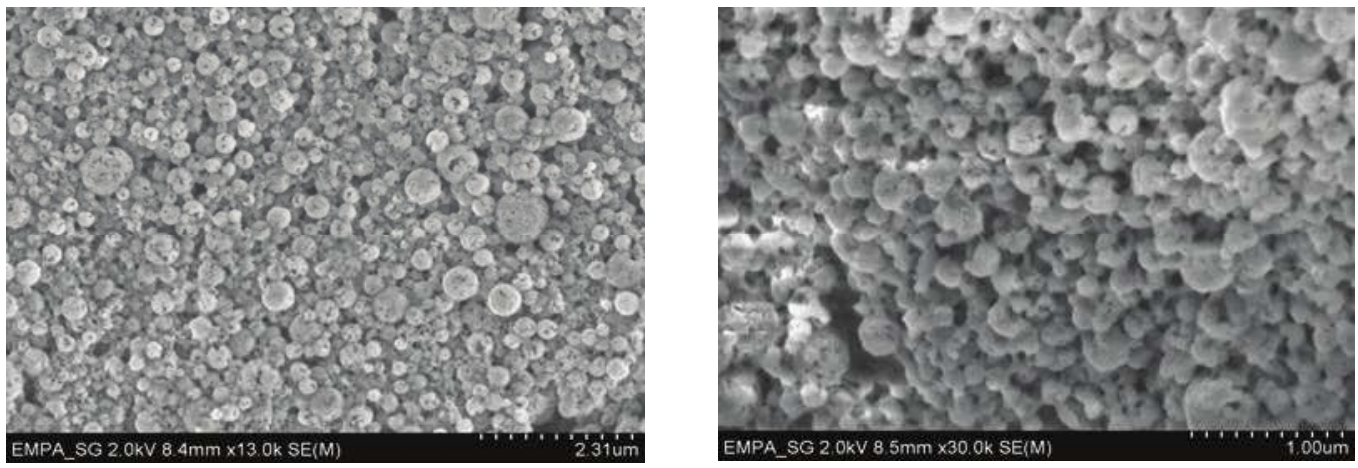

Fig. 5. The SEM images of PDV encapsulated PANI nanoparticles: mp-1 (left) and mp-2 (right).

The amount of PANI base incorporated into PDVB was determined by elemental analysis and found to be $13 \%$ for the first and $15 \%$ for the second polymerization. In order to find out whether core-shell particles had actually formed, XPS measurements were done. Based on nitrogen contents it was concluded that this was not the case but rather many PANI particles were dispersed into larger PDVB particles. The encapsulated particles were purified by dialysis and dried in a vacuum oven at $120{ }^{\circ} \mathrm{C}$. The powder obtained was then ball milled for $10 \mathrm{~min}$. at $20 \mathrm{~Hz}$ using balls of $\varnothing=3 \mathrm{~mm}$. Defined amounts of encapsulated PANI nanoparticles were redispersed both into thermoplastically processed PS- $b$-PB gel and polydimethylsiloxane. For the former composite material it was found that the mechanical properties changed significantly to the worse. For the latter the dielectric properties of prepared films are shown in Figure 6.
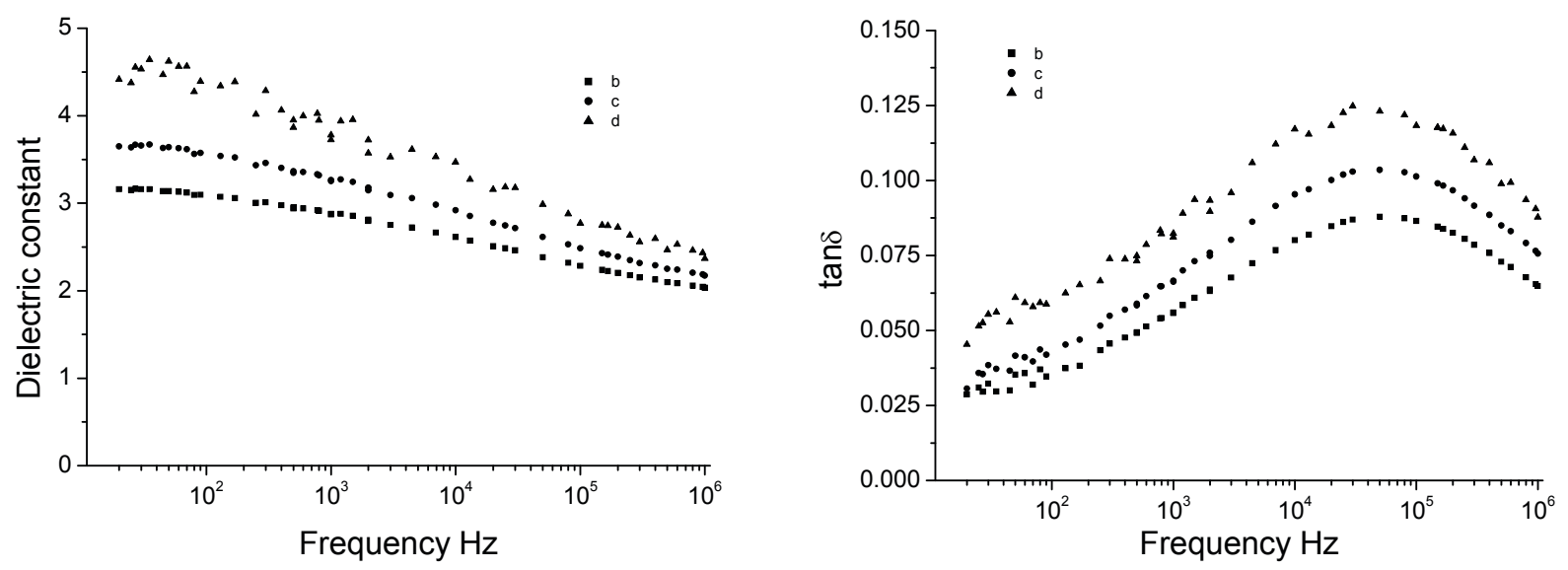

Fig. 6. Dielectric constant and tan $\delta$ as a function of frequency measured for composites with different concentrations of PDVB-encapsulated PANI: $23 \%(\bullet) ; 28 \%(\bullet) ; 33 \%(\boldsymbol{\Delta})$.

The $\varepsilon$ at low frequency for the latter blends increased with increasing amounts of blended particles from $\varepsilon=2.3$ for the pure matrix to almost $\varepsilon=5$. The dielectric loss was less than 0.05 at low frequency. Due to too small weight fractions of PANI in the encapsulated particles, the achievement of a reasonable $\varepsilon$ value of the blends required fractions of encapsulated particles in PDMS too high to ensure good elastic properties. Further work is under way to replace PDVB with a softer PDMS.

\section{ANALYTICAL METHODS}

The particles size was measured using a Beckman Coulter LS 230 instrument and a scanning electron microscope (Hitachi S-4800 instrument). UV-vis absorption spectra were recorded with a Cary 50 spectrophotometer. Dielectric constant measurements were done in the frequency range of $20 \mathrm{~Hz}$ to $1 \mathrm{MHz}$ using an HP 4284A LCR meter. The amplitude of the probing ac electric signal applied to the samples was $1 \mathrm{~V}$. The dielectric constant was determined from the capacitance $C=\varepsilon \varepsilon_{0} A / d$, where $\mathrm{A}$ is the area, $\mathrm{d}$ is the thickness of the capacitor, and $\varepsilon_{0}$ is the vacuum permittivity, 
$\varepsilon_{0}=8.854 \times 10^{-12} C^{2} N^{-1} m^{-2}$. Pellets were prepared by pressing at 9 tons $(\varnothing=13 \mathrm{~mm})$ and were then covered with silver paste electrodes.

\section{MATERIALS}

All chemicals were purchased and used as received. Polystyrene-polybutadiene block copolymer gels was kindly provided by Revoflex. A description of the mechanical properties is given elsewhere. ${ }^{17} \mathrm{Pc}(\mathbf{C})$ was prepared according to literature. ${ }^{7}$ PANI nanoparticles were prepared according to a modified literature procedure. ${ }^{16}$

\section{SYNTHESIS}

\subsection{Preparation of PS-PB-g/PANI composite}

Solutions of appropriate amounts of PANI/DBSA (1:0.5) in toluene (15 wt.\%) were added to a solution of PS- $b$-PB in toluene (10 wt.\%) to obtain five different series of composite with PANI contents from 7 to $32 \mathrm{wt} \%$. The mixtures were stirred for one day followed by sonication for $2 \mathrm{~h}$ in a sonicator bath. Free-standing films were cast by evaporation of toluene at room temperature from the homogeneous solutions and subsequently melt pressed at $100{ }^{\circ} \mathrm{C}$ and $1 \mathrm{t}$. The dielectric properties of the prepared films are shown in figure 4.

\subsection{Synthesis of PANI nanoparticles}

Chemical synthesis in aqueous phase of PANI nanoparticles was based on a literature procedure.16 Poly(styrenesulfonic acid) $\left(M_{w}=80,000 \mathrm{~g} / \mathrm{mol}\right)$ was used as polymeric stabilizer as well as dopant. The PSSA ( $24.22 \mathrm{~g}$ solution $18 \mathrm{wt} \%$ in water), water $(100 \mathrm{ml})$, and aniline $(2.16 \mathrm{~g})$ were mixed together and stirred at $0{ }^{\circ} \mathrm{C}$. To this, a solution of ammonium peroxydisulfate $(2.76 \mathrm{~g})$ in water $(16 \mathrm{~g})$ was added at once. The reaction mixture was stirred overnight and let to warmup to room temperature. After polymerization, the particles were purified by dialysis in water using a cellulose membrane (ZelluTrans Roth, nominal filter rating 3500).

\subsection{Synthesis of encapsulated PANI nanaparticles into PDVB}

Encapsulation of the PANI nanoparticles by direct miniemulsion polymerization of divinyl benzene was achieved using the following procedure. The dispersed phase is an aqueous dispersion of PANI nanoparticles $(6.4 \mathrm{~g})$ containing DVB (the amounts are given in table 1), hexadecane as stabilizer, azobisisobutyronitrile as initiator. The continuous phase was composed of $0.1 \mathrm{~g}$ of surfactant in $18 \mathrm{~g}$ of water. The mixture was stirred for $1 \mathrm{~h}$ and then miniemulsified by ultrasonication for $180 \mathrm{~s}$ with a Sonics sonifier $750 \mathrm{~W}$ Digital at 70\% amplitude. To avoid polymerization due to heating, the mixture was cooled in an ice bath during sonication. The miniemulsion was subsequently transferred to an oil-bath at $75{ }^{\circ} \mathrm{C}$. The aqueous phase containing the particles was washed several times with water by using a membrane. All the miniemulsions were carried out in deionized water. For all miniemulsion experiments, a sonifier (Sonics sonifier $750 \mathrm{~W}$ ) with $13 \mathrm{~mm}$ horn diameter was used.

\subsection{Preparation of PDV encapsulated PANI nanoparticles composite}

A solution of functional PDMS in toluene ( $25 \mathrm{wt} \%$ ) was prepared. To this appropriate amounts of PDVB-encapsulated PANI nanoparticles and cross linker were added to obtain three different series of composite $(23 \%, 28 \%, 33 \%)$. The mixtures were ball milled for $10 \mathrm{~min}$ at $20 \mathrm{~Hz}$ (ball $\varnothing=3 \mathrm{~mm}$ ). Free-standing films were cast by evaporation of toluene at room temperature. The dielectric properties of the prepared films are shown in Figure 6.

\section{CONCLUSION}

We have demonstrated that the dielectric constants for Pcs having carboxylic acid groups strongly depend on the water content. This does not rule out the use of Pcs as fillers for high $\varepsilon$ composite materials, but before this class of substances can be used, fabrication issues of actuators as well as the possible use of imidazole to replace water have to be considered. It was also shown that high $\varepsilon$ values can be achieved when a PS- $g$-PB gel is blended with increasing amounts of PANI. However the blends were too conductive and could not reasonably be used as actuators. Finally, PANI nanoparticles were successfully encapsulated into PDV insulator. According to SEM investigations the average particles size is between 200-280 nm depending on preparation. The dielectric constant at low frequency of PDMS was increased from 2.3 to around 5 when blended with these particles. However, due to the high amount of particles required to achieve this effect, the modulus of the matrix increased. 


\section{ACKNOWLEDGEMENT}

The authors thank Dr. A. Braun and H. Schindler (Empa) for the DLC measurements, P. Rupper and B. Müller for SEM measurements, and the Swiss Federal Institute of Materials Testing and Research (Empa, Dübendorf) for financial support.

\section{REFERENCES}

[1] Bar-Cohen, Y., "Electroactive polymer actuators as artificial muscle”, Proc. SPIE (2001); Ashley, S., "Artificial muscle", Sci. Am. 52, 289, (2003).

[2] Biddiss, E., and Chau, T., "Dielectric elastomers as actuators for upper limb prosthetics: Challenges and opportunities," Med. Eng. Phys. 30(4), 403-418, (2008).

[3] Perline, R. E., Kornbluh, R. D., and Joseph, J. P., "Electrostriction of polymer dielectrics with compliant electrodes as a means of actuation," Sens. Actuators A: Phys. 64(1), 77-85, (1998)

[4] Carpi, F., Gallone, G., Galamtini, F., and De Rossi, D., "Silicone-poly(hexylthiophene) blends as elastomers with enhanced electromechanical transduction properties," Adv. Funct. Mat. 18(2) 235-241, 2008; Huang, C., and Zhang, Q. M., Appl. Phys. Lett. 2004, 84, 22; Zhang, X., Löwe, C., Wissler, M., Jähne, B., and Kovacs, G. "Dielectric elastomers in actuator technology,“ Adv. Eng. Mat. 7(5), 361-367, (2005).

[5] Phougat, N., Vasudevan, P., and Nalwa, H. S., "Handbook of Low and High Dielectric constant Materials and Their Applications"; Volume 1, Chapter 8, Academic Press, (1999); Nalwa, H. S., Dalton, L. R., and Vasudevan, P., "Dielectri properties of copper-phthalocyanine polymer" Eur. Polym. J. 21, 943-947, (1985); Bijwe, J., and Phougat, N., J. Porph. Phthalocyanines, 2, 223, (1998); Bobnar, V., Levstik, A., Huang, C., and Zhang, Q. M., 'Intrinsic dielectric properties and charge transport in oligomers of organic semiconductor copper phthalocyanine," Phys. Rev. B, 71(4), 041202-041206, (2005).

[6] C. Huang, Q. M. Zhang, J. Y. Li, and M. Rabeony, "Colossal dielectric and electromechanical responses in selfassembled polymeric nanocomposites,” Appl. Phys. Lett. 87, 182901-182903, (2005)

[7] Wöhrle, D., and Preussner, E., "Polymeric phthalocyanines and their precursors, 7. Synthesis and analytical characterization of polymers from 1,2,4,5-benzenetetracarboxylic acid derivatives," Makromol. Chem. 186(11), 2189-2207, (1985); Boston, D. R., and Bailar, J. C., "Phthalocyanine derivatives from 1,2,4,5tetracyanobenzene or pyromellitic dianhydride and metal salts," Inorg. Chem. 11(7), 1578-1583, (1972); Achar, B. N., Fohler, G. G., and Parker, J. A., Phthalocyanine polymers. II. Synthesis and characterization of some metal phthalocyanine sheet oligomers," J. Polym. Sci., Polym. Chem. 20, 1785-1790, (1982).

[8] Opris, D. M., Nüesch, F., Löwe, C., Molberg, M., and Nagel, M., "Synthesis, characterization, and dielectric properties of phthalocyanines with ester and carboxylic acid functionalities" Chem. Mater., 20(21), 6889-6896, (2008).

[9] Makarov, S., Litwinski, C., Ermilov, E. A., Suvorova, O., Röder, B., and Wöhrle, D., "Synthesis and Photophysical Properties of Annulated Dinuclear and Trinuclear Phthalocyanines" Chem. Eur. J. 12(5), 14681474, (2006).

[10] A closely related synthetic approach was independently described: Tylleman, B., Gơmez-Aspe, R., Gbabode, G., Geerts, Y. H., and Sergeyev, S. "Ester-functionalized phthalonitriles and zinc phthalocyanines via palladium-catalyzed cyanation of 4,5-dichlorophthalates,"Tetrahedron 64(19), 4155-4161, (2008); Sergeyev,S., Pouzet, E., Debever, O., Levin, J., Gierschner, J., Cornil, J., Gơmez-Aspe, R., and Geerts, Y. H., "Liquid crystalline octaalkoxycarbonyl phthalocyanines: design, synthesis, electronic structure, self-aggregation and mesomorphism," J. Mater. Chem., 17, 1777-1784, (2007); Sergeyev, S., Debever, o., Pouzet, E., and Geerts, Y. H., "Transition temperature engineering of octaalkoxycarbonyl Phthalocyanines," J. Mater. Chem., 17, 30023007, (2007). 
[11] Schuster, M. F. H., Meyer, W. H., Schuster, M., and Kreuer, K. D., "Toward a new type of anhydrous organic proton conductor based on immobilized imidazole," Chem. Mater., 16(2), 329-337, (2004).

[12] Nan, C. W., "Physics of inhomogeneous inorganic materials," Prog. Mater. Sci. 37, 1-116, (1993), Kirkpatrick, S., Rev. Mod. Phys. 45, 574, (1973), Efros, A. L., and Shklovskii, B. I., "Critical behaviour of conductivity and dielectric constant near the metal-non-metal transition threshold," Phys. Stat. Sol. (B), 76(2), 475-485, (1976).

[13] Bodalia, R., Stern, R., Batich, C., and Duran, R., "Synthesis and polymerization of 2-alkylanilines," J. Pol. Sci. Part A Pol. Chem. 31(8), 2123-2127; Cao, Y., Smith, P., and Heeger, A. J. "Counter-ion induced processibility of conducting polyaniline and of conducting polyblends of polyaniline in bulk polymers," Synth. Met. 48(1), 91-97, (1992).

[14] Huang, C., and Zhang, Q. M., "High-dielectric-constant all-polymer percolative composites," Appl. Phys. Lett. 82(20), 3502-3504, (2003).

[15] Volz, M., Ziener, U., Salz, U., Zimmermann, J., and Landfester, K. "Preparation of protected photoinitiator nanodepots by the miniemulsion process," Coll. Polym. Sci. 285, 687-692, (2007); Steiert, N., and Landfester, K. "Encapsulation of organic pigment particles via miniemulsion polymerization," Macromol. Mater. Eng. 292 (10-11), 1111-1125, (2007); Ramirez, L.P., and Landfester, K. "Magnetic polystyrene nanoparticles with a high magnetite content obtained by miniemulsion processes," Macromol. Chem. Phys. 204 (1), 22-31, (2003); Bouanani, R., Bendedouch, D., Hemery, P., and Bounaceur, B. "Encapsulation of montmorillonite in nanoparticles by miniemulsion polymerization," Colloid Surface A, 317, 751-755, (2008); Li, H.L., Li. P., Yang, Y., Qi, W., Sun, H., and Wu, L.X. "Incorporation of polyoxometalates into polystyrene latex by supramolecular encapsulation and miniemulsion polymerization," Macromol. Rapid Commun. 29, 431-436, (2008).

[16] Cho, M. S., Park, S. Y., Hwang, J. Y., and Choi, H. J., "Synthesis and electrical properties of polymer composites with polyaniline nanoparticles," Mat. Sci. Eng., C, 24(1-2), 15-18, (2004).

[17] Opris, D.M., Molberg, M., Löwe, Ch., Nüesch, F., Plummer, C. J. G., Leterrier, Y., and Månson, J.-A. E. Proc. of 9th biennial ASME Conference on Engineering Systems Design and Analysis ESDA, Haifa (2008). 